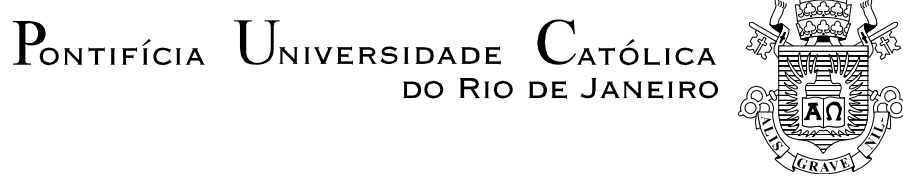

Eduardo Thomaz Faria

\title{
Aplicação de Teoria dos Jogos à Repartição da Energia Firme de um Sistema Hidrelétrico
}

Dissertação apresentada ao Programa de Pós-Graduação em Engenharia Elétrica da PUC-Rio como requisito parcial para obtenção do título de Mestre em Engenharia Elétrica.

Professores Orientadores:

Álvaro Veiga Filho, Docteur Mario Veiga Ferraz Pereira, D.Sc. 


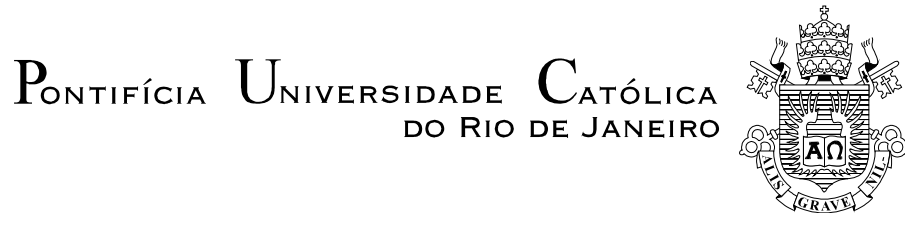

Eduardo Thomaz Faria

\title{
Aplicação de Teoria dos Jogos à Repartição da Energia Firme de um Sistema Hidrelétrico
}

\begin{abstract}
Dissertação de Mestrado apresentada como requisito parcial para obtenção do grau de Mestre pelo Programa de PósGraduação em Engenharia Elétrica do Departamento de Engenharia Elétrica do Centro Técnico Científico da PUC-Rio. Aprovada pela Comissão Examinadora abaixo assinada.
\end{abstract}

\author{
Prof. Álvaro Veiga Filho \\ Orientador \\ Departamento de Engenharia Elétrica - PUC-Rio \\ Dr. Mario Veiga Ferraz Pereira \\ Co-Orientador \\ PSR Consultoria
}

Dr. João Lizardo Rodrigues Hermes de Araújo

UFRJ

Dr. Jerson Kelman ANA

Dr. Sergio Granville PSR Consultoria

Prof. José Eugenio Leal Coordenador Setorial do Centro

Técnico Científico - PUC-Rio

Rio de Janeiro, 21 de maio de 2004 
Todos os direitos reservados. É proibida a reprodução total ou parcial do trabalho sem autorização da universidade, do autor e do orientador.

\section{Eduardo Thomaz Faria}

Graduou-se em Engenharia Elétrica na PUC-RJ em 2001 na área de Sistemas de Apoio à Decisão. Estagiou na empresa Mercados de Energia Ltda., onde participou ativamente de estudos relacionados à avaliação financeira de projetos; comercialização de energia; gerenciamento de risco e otimização físico/financeira para o setor elétrico e estudos de planejamento energético.

Faria, Thomaz Eduardo

Aplicação de teoria dos jogos à repartição da energia firme de um sistema hidrelétrico / Eduardo Thomaz Faria ; orientadores: Álvaro Veiga Filho, Mario Veiga Ferraz Pereira. - Rio de Janeiro : PUC-Rio, Departamento de Engenharia Elétrica, 2004.

167 f. ; $30 \mathrm{~cm}$

Dissertação (mestrado) - Pontifícia Universidade Católica do Rio de Janeiro, Departamento de Engenharia Elétrica.

Inclui referências bibliográficas

1. Engenharia elétrica - Teses. 2. Energia firme. 3. Alocação de custos. 4. Alocação de Benefícios. 5. Teoria dos jogos cooperativos. 6. Otimização linear. 7. Teoria marginalista. I. Veiga Filho, Álvaro. II. Pereira, Mario Veiga Ferraz. III. Pontifícia Universidade Católica do Rio de Janeiro. Departamento de Engenharia Elétrica. IV. Título.

CDD: 621.3 


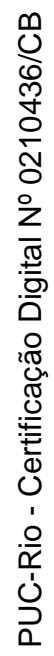

Aos meus pais, Sérgio Nilo e Vera Lúcia.

Aos meus irmãos, Francisco e Fernando. 


\section{Agradecimentos}

Aos meus pais, pela educação, carinho e apoio, sem os quais este trabalho não poderia ter sido concluído.

Aos meus irmãos e família, pelos incentivos demonstrados durante todos os momentos.

Ao amigo Mário Veiga Ferraz Pereira, pela oportunidade de realização deste trabalho e pela orientação indispensável em todas as etapas do desenvolvimento.

Ao amigo Luiz Augusto Barroso, pela excelente orientação e imprescindível disposição em ajudar sempre que foi necessário.

Aos amigos Sérgio Granville e Rafael Kelman, por toda excelente orientação.

Aos amigos Jorge Trinkenreich, Frank Ávila e Priscila Lino, pelos ensinamentos e apoio.

À Cristiane Faria Coelho, pelo carinho e estímulo em todos os momentos.

Ao orientador Álvaro Veiga, pelo estímulo e apoio.

À CNPq e à PUC-Rio, pelos auxílios concedidos

A todos os amigos da PSR/Mercados de Energia. 


\section{Resumo}

Faria, Eduardo Thomaz. Aplicação de Teoria dos Jogos à Repartição da Energia Firme de Um Sistema Hidrelétrico. Rio de Janeiro, 2004, 167 p. Dissertação de Mestrado - Departamento de Engenharia Elétrica, Pontifícia Universidade Católica do Rio de Janeiro.

O objetivo desta monografia é investigar a aplicação de distintas metodologias de alocação de energia firme de usinas hidrelétricas através da teoria dos jogos de coalizão. Mostra-se que não existe uma maneira “ótima”, única, de se fazer esta repartição, mas existem critérios para verificar se uma metodologia de repartição específica apresenta algum aspecto inadequado. Um desses critérios é a "justiça”. Mostra-se que este critério equivale a pertencer ao chamado "núcleo" de um jogo cooperativo. O cálculo da energia firme será formulado como um problema de otimização linear e serão investigadas vantagens e desvantagens de distintos métodos de alocação (a benefícios marginais, geração média no período crítico, última adição e “nucleolus”). Em seguida será desenvolvida uma aplicação do esquema Aumann-Shapley (AS) à repartição da energia firme de usinas hidrelétricas. Demonstra-se que além de robusto em relação aos tamanhos dos recursos e eficiente computacionalmente, este método fornece para o problema do firme uma alocação pertencente ao núcleo e, portanto, atende à condição de “justiça”. A aplicação do esquema AS será apresentada para o Sistema Brasileiro e serão comparados os resultados obtidos por este método com outros esquemas de alocação adotados no Sistema Hidrelétrico Brasileiro.

\section{Palavras-chave}

Engenharia Elétrica, Energia Firme, Alocação de Custos, Alocação de Benefícios, Teoria dos Jogos Cooperativos, Otimização Linear, Teoria Marginalista. 


\begin{abstract}
Faria, Eduardo Thomaz. Allocation of Firm Energy Rights Among hydro plants: a game theoretic approach. Rio de Janeiro, 2004, 167 p. Master Thesis - Electrical Engineering Department, Catholic University of Rio de Janeiro.
\end{abstract}

The objective of this work is to investigate the application of different methodologies of allocation of firm energy rights among hydro plants using a gametheoretic framework. It is shown that there is not an optimal and unique approach to make this allocation but there are criteria to verify if a given approach presents any inadequate aspect. One of these criteria is the "justice", or "fairness". It is shown that this criterion is equivalent to the condition of the core of a cooperative game. The calculation of the firm energy will be formulated as a linear program and advantages/disadvantages of different allocation methods (marginal allocation, average production on the dry period, incremental allocation and "nucleolus") will be investigated. Next, an application of the Aumann-Shapley (AS) scheme to the problem of allocation of firm energy rights will be developed. It is shown that, besides being robust and computationally efficient, this scheme provides an allocation that belongs to the core of the game and therefore meets the condition of "justice". The AS scheme will be applied to the Brazilian system (composed of about 100 hydro plants) and the results obtained will be compared with the allocation schemes currently adopted in the Brazilian system.

\title{
Keywords
}

Electrical Engineering, Firm Energy, Cost Allocation, Benefit Allocation, Cooperative game theory, Linear Optimization, Marginal Theory. 


\section{Sumário}

1 INTRODUÇÃO 13

1.1. Energia firme de uma usina hidrelétrica 13

1.2. Energia firme de múltiplas usinas $\quad 14$

1.3. Sinergia da operação integrada de usinas hidrelétricas 15

1.4. O problema da repartição de benefícios 16

1.5. Objetivos 18

1.6. Organização e principais resultados 18

2 ENERGIA FIRME DE SISTEMAS HIDRELÉTRICOS 22

2.1. Formulação como um problema de otimização - uma única usina 22

2.2. Formulação como um problema de otimização - múltiplas usinas 26

2.3. Aplicações do modelo para cálculo de energia firme. 29

2.4. Período Crítico 34

3 O PROBLEMA DA REPARTIÇÃO DOS BENEFÍCIOS 39

3.1. Teoria de Jogos Cooperativos 39

3.2. Condição para um jogo cooperativo 49

4 ALOCAÇÃO PELA GERAÇÃO MÉDIA NO PERÍODO CRÍTICO (GMPC)59

4.1. Descrição do Método 59

4.2. Vantagens e Desvantagens do Método GMPC 61

5 ALOCAÇÃO A BENEFÍCIOS MARGINAIS (BM) 63

5.1. Descrição do Método 63

5.2. Alocação no Núcleo 64

5.3. Núcleo do Jogo Não Vazio 68 
5.4. Vantagens e Desvantagens do Método

6 ALOCAÇÃO POR ÚLTIMA ADIÇÃO (UA) 70

6.1. Descrição do Método $\quad 70$

6.2. Vantagens e Desvantagens do Método 71

7 ALOCAÇÃO PELO MÉTOdO DO "NUCLEOLUS" 76

7.1. Definição do "Nucleolus" 76

7.2. Descrição do Método do "Nucleolus" 77

7.3. Definição do "Nucleolus" Proporcional 79

7.4. Vantagens e desvantagens do método 81

8 ALOCAÇÃO PELO MÉTODO AUMANN-SHAPLEY (AS) 82

8.1. Método por Benefício Incremental 83

8.2. Método de Shapley 83

8.3. Método Aumann-Shapley (AS) 85

8.4. Alocação no Núcleo $\quad 89$

8.5. Vantagens e Desvantagens do Método AS 91

9 RESULTADOS DA APLICAÇÃO DOS MÉTODOS DE ALOCAÇÃO 93

9.1. Resultados da aplicação a três sistemas-exemplo fictícios 93

9.2. Resultados: Sistema Interligado Brasileiro 100

9.3. Alocação da Energia Firme do Sistema Interligado Brasileiro 101

9.4. Resultados por cascata 107

9.5. Análise dos resultados: síntese geral 114

10 CONCLUSÕES 116

11 REFERÊNCIAS 119

12 ANEXO A - MÉTODO DO DIAGRAMA DE MASSAS (ou DIAGRAMA DE RIPPL). 127 
13 ANEXO B - ENERGIA ASSEGURADA E O SISTEMA BRASILEIRO131

13.1. Certificados de Energia Assegurada (CEA) 131

13.2. Importância comercial dos CEAs 132

13.3. Cálculo dos CEAs - Sistema 100\% hidroelétrico 132

13.4. Cálculo dos CEAs - sistema hidrotérmico 136

13.5. Outros Temas para Discussão na metodologia atual 139

14 ANEXO C -O MECANISMO DE REALOCAÇÃO DE ENERGIA 143

14.1. MRE: Motivação 143

14.2. O Mecanismo de Realocação de Energia 145

14.3. Exemplo de Aplicação do MRE 146

14.4. Vantagens e Limitações do MRE 151

15 ANEXO D - MÉTODO AUMANN-SHAPLEY: SEÇÕES AUXILIARES153

15.1. Efeito da ordem de entrada na alocação por benefício incremental153 15.2. Efeito do tamanho dos agentes no método de Shapley 155 15.3. Desenvolvimento do método AS a partir do método de Shapley: uma interpretação intuitiva. 157

16 Anexo E - Certificados de energia assegurada das usinas do Sistema Interligado Brasileiro. 


\section{Lista de figuras}

Figura 1.1 - Gráfico de sucessivas aplicações do método de Rippl 14

Figura 2.1 - Resultados do cálculo da energia firme para 1 usina 31

Figura 2.2- Legenda das figuras usadas para representar usinas hidrelétricas 32

Figura 2.3 - Topologia dosistema-exemplo 32

Figura 2.4 - Resultados do cálculo da energia firme para 4 usinas 34

Figura 3.1 - Representação geométrica do núcleo 49

Figura 3.2 - Usinas em paralelo $\quad 54$

Figura 3.3- Usinas em série $\quad 54$

Figura 3.4 - Nova representação das usinas em série 56

Figura 5.1 - Exemplo do efeito do aumento do reservatório no método BM 69

$\begin{array}{ll}\text { Figura 9.2 - Cascata do rio Iguaçu } & 109\end{array}$

Figura 9.3 - Resultados dos três métodos de alocação das usinas do rio Iguaçu 109

Figura 9.4 - Cascata do rio Tocantins 110

Figura 9.5 - Resultados dos três métodos de alocação das usinas do rio Tocantins 110

Figura 9.6 - Cascata do rio São Francisco 111

Figura 9.7 - Resultados dos três métodos de alocação das usinas do rio Tocantins 112

Figura 9.8 - Cascata do rio Paraná e seus afluentes 113

Figura 9.9 - Resultados dos três métodos de alocação das usinas do rio Paraná e dos valores agregados das usinas de seus rios afluentes. 


\section{Lista de tabelas}

Tabela 2.1 - Características físicas da usina do primeiro exemplo 30

Tabela 2.2 - Características físicas das 4 usinas segundo exemplo 33

Tabela 3.1 - Possíveis alocações de Energia Firme das usinas 48

Tabela 9.1 - Sistemas-exemplo 94

Figura 9.1- Topologia das cascatas dos sistemas-exemplo 94

Tabela 9.2 - Resultados dos quatro métodos de alocação aplicados ao CASO 1

Tabela 9.3 - Resultados dos quatro métodos de alocação aplicados ao CASO 2

Tabela 9.4 - Resultados dos quatro métodos de alocação aplicados ao CASO $3 \quad 99$

Tabela 9.5 - Resultados dos métodos GMPC, UA, AS aplicados ao sistema hidrelétrico brasileiro e seus respectivos Certificados de Energia Assegurada 106 